Cahiers

d'ethnomusicologie

\section{Cahiers d'ethnomusicologie}

Anciennement Cahiers de musiques traditionnelles

17 | 2004

Formes musicales

\title{
Uruguay: un mapa musical del Uruguay (Lauro Ayestarán)
}

Compilé d'après des copies d'originaux des archives sonores du musicologue Lauro Ayestarán, 2003

Ignacio Cardoso Silva

\section{(2) OpenEdition}

\section{Journals}

Édition électronique

URL : http://journals.openedition.org/ethnomusicologie/542

ISSN : 2235-7688

Éditeur

ADEM - Ateliers d'ethnomusicologie

Édition imprimée

Date de publication : 1 janvier 2004

Pagination : 374-376

ISBN : 2-8257-0910-7

ISSN : $1662-372 X$

\section{Référence électronique}

Ignacio Cardoso Silva, «Uruguay: un mapa musical del Uruguay (Lauro Ayestarán) », Cahiers

d'ethnomusicologie [En ligne], 17 | 2004, mis en ligne le 13 janvier 2012, consulté le 01 mai 2019. URL http://journals.openedition.org/ethnomusicologie/542

Ce document a été généré automatiquement le 1 mai 2019.

Tous droits réservés 


\title{
Uruguay: un mapa musical del Uruguay (Lauro Ayestarán)
}

Compilé d'après des copies d'originaux des archives sonores du musicologue Lauro Ayestarán, 2003

\author{
Ignacio Cardoso Silva
}

\section{RÉFÉRENCE}

Lauro Ayestarán, Uruguay: un mapa musical del Uruguay, Compilé d'après des copies d'originaux des archives sonores du musicologue Lauro Ayestarán, propriété de son disciple et musicologue Coriún Aharonián qui a également coordonné la réalisation de ce CD. Livret avec textes et photos 20p. Digitalisation et enregistrement: Ediciones Tacuabé, Studio Elac, Montevideo, Uruguay. Production: Ediciones Tacuabé. 1 CD Ayuí - Serie de la Memoria - A/M 38 CD, 2003.

1 Voici le premier disque de ce qui devrait être une grande collection chez Ayuí/Tacuabé dédiée à l'œuvre essentielle du musicologue uruguayen Lauro Ayestarán. Il inaugure la mise en valeur tant attendue des archives sonores constituées par le musicologue entre 1943 et 1966 et qui représentent plus de 4000 enregistrements réalisés dans tout le pays par lui et ses assistants. Comme son titre l'indique, le CD présente une carte musicale de la musique traditionnelle uruguayenne, reflet de plusieurs décennies de travail de terrain.

2 Lauro Ayestarán figure parmi les plus grands musicologues latino-américains. Son labeur gigantesque est à l'origine de pratiquement toutes les études musicologiques et ethnomusicologiques réalisées en Uruguay postérieurement à sa mort prématurée en 1966. Il est le chercheur qui a contribué à faire entrer les enquêtes sur le folklore dans les rangs des disciplines académiques. Il a facilité une prise de conscience sur la diversité culturelle de la société uruguayenne et a dressé un profil musicologique extrêmement complet du pays. Né à Montevideo en 1913, il poursuivra des études de piano et de théorie 
musicale jusqu'en 1934. A vingt ans, il devient critique de musique, théâtre, littérature et cinéma. Son premier intérêt pour les enquêtes musicologiques se porte vers la musique classique européenne et américaine. Cependant, il se trouvera face à de nombreuses références à la musique autochtone et aux réinterprétations américaines des musiques de salon venues d'Europe. C'est ce qui le poussera dès lors à rechercher les origines de la musique sur le territoire uruguayen. Il s'intéressera en particulier aux transformations et métissages que subissent les courants musicaux majeurs du continent, c'est-à-dire aux apports des musiques préhispaniques, africaines et européennes auxquels il voue un intérêt égal. Ce sera le travail de toute une vie puisque qu'en 1953 il publie le premier et, malheureusement, unique des trois tomes prévus sur la musique en Uruguay ${ }^{1}$. Le livre comporte tout de même plus de 800 pages, il couvre quasiment tous les genres musicaux, des témoignages de musiques préhispaniques aux expressions rurales et urbaines du $\mathrm{XX}^{\mathrm{e}}$ siècle. La publication a tout de l'ouvrage encyclopédique, rappelant par là l'œuvre de son contemporain cubain Fernando Ortíz. Sa méthode est novatrice. Il compile et épluche systématiquement toutes les références écrites: récits de voyages, archives ou partitions anciennes, et les confronte aux matériaux des autres pays de la région. Il intègre également un formidable travail de terrain où il interroge les sources orales et enregistre quantité de documents sonores. En avance sur son temps, Ayestarán n'en subit pas moins les influences des paradigmes anthropologiques dominants de l'époque. Ses études sur le «fait folklorique national» font appel à la notion de survivance et ont tendance à renvoyer à une reconstruction de formes passées à partir desquelles les formes actuelles sont évaluées en termes de "pur et impur». Ce point de vue renforçait l'idée que le folklore en tant que survivance était condamné à disparaître avec le temps et le progrès de la civilisation, induisant une conception unilinéaire voire évolutionniste du changement social.

3 En plus de ses activités de journaliste et de chercheur, il assurait le poste de professeur de musicologie à la Facultad de Humanidades y Ciencias de l'Université de Montevideo, ainsi qu'à l'Instituto de Profesores Artigas, l'Escuela Municipal de Música et au Conservatorio Nacional où il enseignait, outre la musicologie, l'histoire de la musique. Enfin, il était directeur de programmation à la radio d'Etat, le SODRE. Ce travailleur acharné a produit plus de cent monographies, publié une vingtaine de livres et constitué les fonds sonores qui font aujourd'hui l'objet de cette première édition destinée au grand public. Lauro Ayestarán décède à l'âge de 53 ans sans pouvoir atteindre tous les objectifs qu'il s'était fixés, laissant derrière lui toute une série de travaux inachevés qui seront en partie complétés et publiés par les membres de sa famille.

Ce $\mathrm{CD}$ contient des pièces représentatives des musiques traditionnelles et populaires d'Uruguay. Les enregistrements de terrain ont été effectués dans un premier temps sur disque d'acétate, puis ultérieurement sur bandes magnétiques, au moyen d'un enregistreur à bandes portable Butoba et, quand les circonstances le permettaient, sur support fixe en studio. Les 31 plages du disque font la part belle aux musiques et danses rurales. Les styles musicaux estilo (ou triste), milonga, cifra et vidalita se caractérisent par du chant accompagné à la guitare dont les paroles évoquent parfois les guerres civiles qui ont maintes fois ravagé les campagnes uruguayennes, comme c'est le cas de la milonga La Revolución de 1910 (plage 5), interprétée en 1957 par Juan García qui était aveugle, et de la vidalita Los Tres Valientes (La Revolución de 1897), plage 11, interpétée par le payador (chanteur gaucho) Aníbal Sampayo. El estilo est un genre rural ancien que l'on joue peu fréquemment aujourd'hui, contrairement à la milonga. On remarquera la plage 3, Madre, 
pièce anonyme interprétée par Ramón López qui nous parle d'un fils plein de remords revenant s'occuper de sa mère en fin de vie, après l'avoir abandonnée dans sa jeunesse. La plage 8 nous présente une Cifra, El Facón brasilero, une improvisation du payador Juan Pedro López qui traite avec humour et finesse des histoires de bagarres entre gauchos.

Le disque présente également plusieurs danses comme la Polca, la Mazurca, el Pericón ou la Chimarrita. Ces pièces comprennent presque toutes un accordéon accompagné de chant. Ces styles sont de plus en plus rares de nos jours. Il faut relever que les genres ruraux de musique et de danse sont des traditions partagées avec les régions frontalières des pays voisins (Brésil, Argentine). Notons la présence de deux tangos (plages 20 et 21), dont le premier est exécuté en 1943 par Policarpo Pereira, un afro-uruguayen de Montevideo avec un arc en bouche uricungo. Il est peut-être le dernier à avoir maintenu cet instrument vivant en Uruguay.

6 L'auditeur prendra plaisir à écouter quatre fabuleux enregistrements de candombe afrouruguayen réalisés à Montevideo entre 1958 et 1966 (plages 22 à 25), dont un (plage 22) durant le carnaval de 1958. On appréciera tout particulièrement l'intention de recueillir précisément le jeu de chaque tambour. Le connaisseur reconnaîtra un style «à l'ancienne» avec la présence du bombo ou bajo, gros tambour basse, aujourd'hui de moins en moins utilisé. On reconnaît également la voix de son assistant Coriún Aharonián qui possède en copies et originaux la quasi-totalité des fonds sonores d'Ayestarán. Les deux dernières plages dédiées au candombe ont été enregistrées dans des conventillos, hauts lieux de regroupement, de sociabilité et de transmission culturelle des Afro-Uruguayens, qui ont été détruits durant la période de dictature militaire (1973-1984).

7 Un des derniers genres représentés dans ce disque est la murga (plage 26),genre carnavalesque par excellence, très populaire, qui consiste en un grand chœur d'hommes accompagné d'une section de tambours de fanfare (grosse caisse et caisse claire) et de cymbales. L'enregistrement date de 1946 et atteste également d'un style qui a énormément évolué depuis.

Enfin, le disque se termine par une série d'enregistrements témoignant de l'ouverture d'esprit et de la globalité du travail d'Ayestarán. Les «sons» de la ville de Montevideo sont présents à travers deux pregones (cris de rue) qui font encore partie de l'univers sonore urbain, celui d'un heladero (vendeur de glace) et celui de l'afilador (rémouleur). On trouve pour finir un chant religieux en portuñol, dialecte du nord de l'Uruguay (plage 29), un chant pour enfant, romance (plage 30), et une ronde ou comptine traditionnelle d'enfants, ronda (plage 31), enregistrée dans la ville frontalière uruguayo-brésilienne de Rivera en 1958.

9 Un mot encore sur la maison de disque Ayuí/Tacuabé. Crée en 1971, entre autres par Coriún Aharonián, elle travaille pour la diffusion de la musique uruguayenne traditionnelle, populaire et moderne, à l'encontre de « la logique de marché». Son action, souvent limitée par des obstacles politiques et économiques, a contribué à développer et soutenir la très riche et variée musique populaire uruguayenne ${ }^{2}$.

10 Ce CD suscite l'espoir de voir peut-être enfin publiée la grande collection de Lauro Ayestarán, chercheur infatigable, afin que son œuvre gigantesque soit accessible à tous et qu'elle transcende les frontières de ce petit pays latino-américain. 


\section{NOTES}

1. La Música en el Uruguay. Tomo I. Montevideo : Sodre, 1953

2. Un catalogue est consultable sur le web à l'adresse suivante : http://www.tacuabe.com 